\title{
ist \\ Racionalidade, consistência, reticulação e coerência: o caso da renormalização na teoria quântica do campo
}

Valter Alnis Bezerra

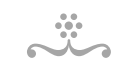

RESUMO

Um exame do desenvolvimento da teoria quântica do campo, no período que vai de 1927 a 1971, evidencia o fato de que as técnicas de renormalização desempenharam um papel fundamental ao longo de todo o processo. Em particular, a renormalização reabilitou a eletrodinâmica quântica no final dos anos 4,0 e, depois, também reabilitou a teoria quântica do campo como um todo e ajudou a consolidar as teorias de gauge no início dos anos 7̧o. $\mathrm{O}$ sucesso da renormalização foi tal que ela passou gradualmente de mero dispositivo teórico $a d$ hoc à condição de critério para a construção e avaliação de teorias na física do campo. Um dos objetivos deste artigo é mostrar que essa transformação no estatuto metodológico da renormalização pode ser entendida no contexto do modelo reticulado de racionalidade de Larry Laudan. Apesar do extraordinário progresso teórico e empírico alcançado, porém, sempre houve polêmicas acerca do lugar que a renormalização deve ocupar dentro da estrutura conceitual da disciplina. Essas polêmicas giram em torno do fato de que a renormalização aparentemente vai contra um valor cognitivo considerado fundamental: a consistência. O segundo objetivo deste artigo é mostrar como o modelo reticulado também permite lançar luz sobre essa questão. Procuramos expor em que sentido foi racional adotar a renormalização na física de partículas e campos apesar de existir o problema da inconsistência. Para isso nos valemos das teses do modelo reticulado, complementadas por teses de Putnam, Quine e da teoria coerencial da justificação.

PALAVRAS-CHAVE $\bullet$ Metodologia científica. Racionalidade científica. Modelo reticulado.

Valores cognitivos. Teoria quântica do campo. Teoria de gauge. Renormalização. Inconsistência.

\section{INTRODUÇÃ̃o}

A teoria quântica do campo constitui um programa de pesquisa extremamente bemsucedido dentro da física do século XX. Nascido dos esforços para descrever sob um ponto de vista quântico a interação eletromagnética, através da eletrodinâmica quântica, o programa da teoria quântica do campo foi gradualmente estendido com sucesso aos outros tipos de interações existentes na natureza - com exceção, por enquanto, da 
gravitação - e acumulou um notável cabedal de êxitos empíricos. Porém a teoria quântica do campo se viu desde o início assolada por um problema sério, que é o problema das divergências. Em várias situações, a teoria prevê valores infinitos para diversas quantidades que deveriam ser observáveis. Neste artigo iremos discutir dois casos historicamente importantes nos quais o problema se manifestou: o primeiro é o dos infinitos na eletrodinâmica quântica, e o segundo é o das divergências na classe de teorias conhecidas como teorias de gauge não-abelianas. Em ambos os casos, a teoria quântica do campo se defrontou com difíceis impasses. Também em ambos os casos, a chave para solucionar o problema estava num procedimento conhecido como renormalização, que será o objeto de nosso interesse neste texto.

Estamos interessados em analisar a racionalidade do procedimento de renormalização, e para isso nos valeremos do modelo reticulado de racionalidade científica proposto por Larry Laudan. O modelo reticulado prevê, entre outras coisas, a mutabilidade da metodologia e da axiologia científica, e a interação não-hierárquica entre os componentes da estrutura cognitiva de uma disciplina. Uma análise reticulacional permite colocar em perspectiva alguns aspectos importantes da aceitação da renormalização. Primeiro, ela permite apreciar a relação de condicionamento recíproco que existe entre teorias científicas e metodologias. Em particular, podemos apreciar como a renormalização passou de um dispositivo ad hoc das teorias a um critério metodológico fundamental da física do campo, e isso basicamente em decorrência de uma pressão gerada pelo desempenho, na prática, de uma certa classe de teorias. Em segundo lugar, a visão reticulacional pode nos ajudar a compreender em que sentido a aceitação da renormalização pelos físicos pode ser considerada racional, a despeito das polêmicas que cercaram (e ainda cercam) a sua suposta inconsistência. Para tentar esclarecer essa questão, valemo-nos das teses do modelo reticulado, complementadas por uma visão coerencial de justificação e por teses de Putnam, sobre o caráter empírico da lógica, e de Quine, sobre o holismo teórico.

\section{BREve históRIA DA TEORIA QUÂNTICA DO GAMPO}

A teoria quântica do campo possui características bastante diferentes da mecânica quântica usual de partículas. Pode-se dizer que a teoria quântica do campo nasce de uma tripla convergência - entre a teoria lagrangiana do campo, a mecânica quântica e a relatividade restrita - com o objetivo de descrever os campos, entendidos como sistemas com um número infinito de graus de liberdade. A primeira tentativa de estender a mecânica quântica aos campos remonta já aos primeiros anos da teoria, mais precisamente a 1926, quando Born, Heisenberg e Jordan descreveram o campo eletromagné- 
tico no vácuo (isto é, na ausência de cargas e correntes) como um sistema de osciladores, concluindo que a energia do campo seria quantizada (Born, Heisenberg \& Jordan, 1968). Em 1927, Paul Dirac criou o chamado método da segunda quantização (Dirac, 1927) e formulou a primeira teoria quântica do campo, a eletrodinâmica quântica, que lhe permitiu tratar o problema da emissão e absorção de radiação, um processo no qual partículas são criadas e destruídas (os fótons). Jordan \& Wigner (1928) estenderam o método da segunda quantização (inicialmente formulado para bósons, isto é, partículas com spin inteiro) aos férmions (partículas com spin semi-inteiro), e Jordan \& Pauli (1928) tornaram o método relativístico. Os anos 3o foram um período de grande desenvolvimento para a teoria quântica do campo. Ao final da década, os conceitos fundamentais da teoria já se encontravam bem estabelecidos no arsenal conceitual dos físicos. 'Houve inclusive tentativas de se estender o programa da teoria quântica do campo a outras interações, além da eletromagnética - em particular a interação nuclear fraca, por Fermi, e a interação nuclear forte, por Yukawa. ${ }^{2}$

Historicamente, foi na eletrodinâmica quântica que o problema das divergências apareceu pela primeira vez, e também foi no contexto da eletrodinâmica que surgiu a renormalização. ${ }^{3}$ Uma forma do problema é a chamada "catástrofe ultravioleta" que aparece ao se calcular os efeitos da auto-energia do elétron e da polarização do vácuo. Ambos os efeitos estão relacionados com a criação de partículas virtuais a partir do vácuo, o qual, na teoria quântica do campo, possui um caráter dinâmico. A auto-energia do elétron resulta do fato de que existe uma contínua emissão e reabsorção de fótons virtuais por qualquer carga elétrica. Assim o elétron tem que interagir com uma "nuvem" de fótons virtuais produzidos por ele mesmo - ou, em outras palavras, interage com o seu próprio campo. Essa auto-interação tem o efeito de alterar a energia do elétron, ou, de acordo com a equivalência massa-energia, a sua inércia efetiva. Já na

\footnotetext{
1 Entre os conceitos fundamentais do programa da teoria quântica do campo, podemos citar: (a) a descrição dos campos em termos de operadores, em vez de funções ou distribuições; (b) o conceito de criação e destruição de partículas, descrito pelos operadores de criação e aniquilação; (c) a quantização da energia dos campos; (d) o papel crucial desempenhado pelo estado do vácuo, como estado fundamental sobre o qual atuam os operadores de criação, para construir recursivamente os outros estados; (e) o conceito de antipartícula; (f) o conceito de partículas virtuais; (g) a interpretação das forças ou interações como processos de troca de partículas; (h) o caráter local das interações; (i) a conexão entre spin e estatística.

2 A interação nuclear fraca é responsável pelo decaimento beta, e a interação forte é responsável pela coesão do núcleo atômico, contrabalançando a repulsão eletrostática entre os prótons.

30 primeiro e mais elementar tipo de divergência que surge dentro da teoria dizia respeito à previsão de uma energia infinita para o estado de vácuo. Nesse caso, raciocinou-se que, como são experimentalmente detectáveis apenas as diferenças entre energia, porém não as energias absolutas, bastaria redefinir a escala de energia, e medir todas as energias relativamente ao estado de vácuo. Isso é implementado na prática por meio de um truque matemático conhecido como ordenamento normal de operadores, onde se estipula que certos comutadores devem se anular.
} 
polarização do vácuo, o campo eletrostático de um elétron leva ao surgimento de pares elétron-pósitron virtuais que são criados a partir do vácuo. Os elétrons virtuais são repelidos pela carga negativa original, ao passo que os pósitrons são atraídos. Por causa dessa assimetria, o elétron original fica envolvido por uma nuvem de pósitrons virtuais que blinda parte da sua carga, resultando em uma carga efetiva que é menor do que a carga original. O vácuo se comporta, assim, como um meio dielétrico polarizável pelo campo elétrico, daí o nome do efeito.

O grande problema é que ambos os efeitos, quando calculados na teoria do campo, dão origem a resultados que divergem, isto é, tendem ao infinito, quando se leva em conta a contribuição devida às partículas com momentos arbitrariamente altos. ${ }^{4}$ (Momentos elevados correspondem, no contexto da dualidade partícula-onda, a baixos comprimentos de onda, como na radiação ultravioleta, daí o nome de "catástrofe ultravioleta".) O problema com a auto-energia do elétron foi detectado por Oppenheimer (193oa) e analisado por Weisskopf (em 1934 e 1939; respectivamente, Weisskopf, 1994a; 1939). O problema com a polarização do vácuo também foi identificado por Oppenheimer (193ob) e analisado por Dirac (em 1933 e 1934; respectivamente, Dirac, 1994a; 1994b), Furry \& Oppenheimer (1934), Heisenberg (em 1934; ver Heisenberg, 1994) e Weisskopf (em 1936; ver Weisskopf, 1994b). As divergências da eletrodinâmica quântica levavam a previsões absurdas para resultados experimentais, tais como o espaçamento das linhas espectrais e as seções de choque de espalhamento. ${ }^{5}$

Nos anos $3 \circ$ e 4. 0 , diversas técnicas foram desenvolvidas para eliminar ou pelo menos contornar os infinitos da teoria quântica do campo, como os "campos compensadores" e a chamada "física da subtração". Todas essas técnicas pioneiras, porém, possuíam um caráter ad hoc e ainda não estavam integradas numa abordagem sistemática. Além disso, elas envolviam certas operações com quantidades infinitas que eram difíceis de justificar rigorosamente em termos matemáticos. Por isso, a atitude que reinava nos anos 4.0 entre os físicos era basicamente a de continuar usando a teoria quântica do campo, na falta de outra abordagem melhor, porém com uma ponta de ceticismo.

\footnotetext{
4 Em termos matemáticos, levar em conta momentos arbitrariamente altos significa calcular integrais impróprias, ou seja, integrais nas quais um dos limites de integração, ou ambos, são infinitos. Apesar de serem definidas sobre um intervalo infinito, as integrais impróprias comumente convergem, isto é, possuem valor finito. Porém as integrais importantes no caso da auto-energia do elétron e da polarização do vácuo são divergentes.

5 Um outro tipo importante de divergência era a "catástrofe infravermelha", decorrente de processos envolvendo fótons com momento baixo (ou, equivalentemente, comprimento de onda alto, como na radiação infravermelha). Esse tipo de divergência produzia resultados infinitos para as seções de choque, o que invalidava certas previsões concernentes aos problemas de espalhamento. Entre os que estudaram o problema estão Pauli e Fierz (em 1938; ver Pauli \& Fierz, 1994) e Dancoff (1939).
} 
Ragionalidade, GonsistênGia, Retigulação e goerência...

A solução para o problema foi encontrada nas técnicas de renormalização. O conceito moderno de renormalização baseia-se na idéia de que a massa efetiva de um elétron, por exemplo, deve ser entendida como formada por dois componentes: uma massa "limpa" (sem a presença de fótons virtuais), que se pressupõe ser infinita, e também uma "auto" - massa (resultante dos fótons virtuais), que pode ser calculada na teoria, dando um resultado infinito. A idéia é que uma quantidade infinita "cancela" a outra, num certo sentido preciso, resultando num valor finito que coincide com o valor observado experimentalmente. O fato de que a massa "limpa" é infinita não deve constituir problema, uma vez que ela não pode ser observada diretamente. A renormalização é, portanto, um processo de eliminar os infinitos absorvendo-os dentro de uma redefinição dos parâmetros físicos (Schwinger, 1958, p. xi; Weinberg, 1977, p. 27). O processo pode ser aplicado a outros parâmetros além da massa, como por exemplo a carga.

O procedimento de renormalização não deve ser considerado como apenas mais um exemplo da "física da subtração" mencionada acima. Evidentemente, a "subtração" de uma quantidade infinita de outra quantidade também infinita não parece ser uma operação matematicamente bem definida. Na prática, a redefinição dos parâmetros é obtida formalmente por um processo de "corte" (em inglês cutoff) nas integrais relevantes e subseqüente passagem ao limite. ${ }^{6}$ Porém, restava o problema de como executar esse cutoff de forma a preservar a covariância relativística.

A noção de renormalização já havia sido sugerida por Weisskopf (em 1936; ver Weisskopf, 1994b) e por Kramers (1938), porém não atraiu grande interesse no início. Os físicos percebiam que, para um tratamento sistemático da questão, três coisas ainda eram necessárias. Primeiro, era preciso encontrar uma maneira de classificar os tipos de infinitos que surgiam nos cálculos, uma vez que, para que a técnica de renormalização possa ser aplicada, os infinitos precisam aparecer de uma maneira específica, isto, é, na forma de correções aos parâmetros. Segundo, era indispensável descobrir como executar o procedimento de renormalização de tal forma que não se destruísse a covariância relativística. Terceiro, faltava uma quantidade maior de dados experimentais, capazes de permitir uma discriminação fina entre as várias técnicas de eliminação de infinitos. Todos esses três elementos iriam emergir de maneira dramática no final dos anos 40 .

6 Esse "corte" nas integrais sobre os momentos pode ser executado, seja especificando um valor grande, porém finito, como limite superior de integração, seja multiplicando o integrando por uma função apropriada, que cai próxima de zero para momentos acima de um certo valor. O importante é fazer com que os termos que dependem do limite superior ou da função escolhida sejam precisamente os termos que têm problemas de divergência, enquanto os outros termos permanecem quase independentes dessa escolha. 
Num experimento que se tornaria célebre, Lamb e Retherford realizaram uma medida de precisão do desdobramento hiperfino dos níveis de energia do átomo de hidrogênio (Lamb \& Retherford, 1947). De acordo com a teoria de Dirac do elétron, de 1928, as energias dos dois primeiros estados excitados do hidrogênio deveriam ser iguais, porém Lamb e Retherford mostraram que eles diferiam por $1000 \mathrm{MHz}$, um efeito que ficaria conhecido como "deslocamento de Lamb" (Lamb shift). Esse resultado foi anunciado no famoso congresso de física de Shelter Island em 1947. Imediatamente em seguida ao congresso, Hans Bethe (1947) formulou uma explicação para o deslocamento de Lamb em termos da auto-interação do elétron, usou a renormalização da massa para eliminar as divergências, e encontrou um valor próximo ao observado. Porém, assim como os outros cálculos de renormalização efetuados na eletrodinâmica de então, o cálculo de Bethe não possuía covariância relativística.

Parcialmente impulsionados pelos acontecimentos de Shelter Island, Richard Feynman e Julian Schwinger deram entre 1947 e 1949 os toques finais numa eletrodinâmica quântica de caráter sistemático, que era ao mesmo tempo renormalizável e covariante (cf. Schwinger, 1948; 1949a; 1949b; Feynman, 1948a; 1948b; 1949a; 1949b). Sin-Itiro Tomonaga já havia desenvolvido no Japão uma teoria desse tipo em 194,3, publicada em inglês em 1946 (cf. Tomonaga, 1946; 1948). Em seguida, Freeman Dyson (1949a; 1949b) demonstrou que os formalismos de Feynman, Schwinger e Tomonaga eram equivalentes, e conseguiu fornecer uma classificação dos tipos de divergências da eletrodinâmica quântica, provando que elas eram precisamente do tipo que poderia ser removido através da renormalização.

Nos anos que se seguiram, a eletrodinâmica quântica provocou um grande entusiasmo entre os físicos, e os cálculos foram sendo executados com aproximação cada vez melhor. Atualmente, os cálculos mais detalhados efetuados utilizando a teoria conseguem atingir uma impressionante precisão de dez casas decimais (ver Mandl \& Shaw, 1993). O sucesso da eletrodinâmica quântica inspirou na comunidade científica a esperança de que as outras interações da Natureza, além da eletromagnética, pudessem ser descritas por meio de teorias quânticas renormalizáveis.

Porém, à medida que se prosseguia nesse projeto, percebeu-se que isso dificilmente seria conseguido no que se refere à interação nuclear fraca e à interação forte. No caso da interação fraca, as divergências que surgiam eram de um tipo que não podia ser erradicado por meio das técnicas de renormalização existentes. No caso da interação forte, era impossível aplicar a teoria da perturbação - que era o modo pelo qual a maioria dos cálculos eram efetuados na teoria do campo - devido ao valor elevado da constante de acoplamento. ${ }^{7}$ Essas dificuldades levaram o programa da teoria quântica do campo a um impasse em meados dos anos 5 o. Esse impasse provocaria o abandono do projeto por grande parte da comunidade científica, que se voltou então para outras abor- 


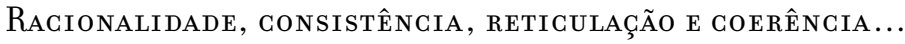

dagens, como o programa das simetrias e da álgebra de correntes, ou então para o programa da matriz-S. Essa situação persistiria até o final dos anos 60.

\section{Quadro 1. CRonologia resumida da eLETrodinÂMiGa quÂnTIGA}

1926: Born, Heisenberg e Jordan - Primeira descrição do campo eletromagnético no vácuo como um sistema de osciladores

1927: Dirac - Método da segunda quantização; eletrodinâmica quântica; tratamento do problema da emissão e absorção de radiação

1928: Jordan, Wigner, Pauli - Versão relativística do método da segunda quantização; extensão ao caso dos férmions

Anos 3o: Fermi, Yukawa - Tentativas de estender o programa da teoria quântica do campo à interação nuclear fraca e à interação nuclear forte

1930: Oppenheimer - Identificação dos problemas da auto-energia do elétron e da polarização do vácuo

1934, 1939: Weisskopf - Análise do problema da auto-energia do elétron

1933, 1934, 1936: Dirac, Furry-Oppenheimer, Heisenberg, Weisskopf - Análise do problema da polarização do vácuo

Anos 3o e 40: Técnicas ad hoc para eliminar os infinitos na teoria quântica do campo; ceticismo em relação à teoria quântica do campo

1936, 1938: Weisskopf, Kramers - Primeiras sugestões do conceito moderno de renormalização

7 Na teoria da perturbação, os cálculos são efetuados calculando inicialmente um termo básico que possui forma relativamente simples, sem considerar os detalhes do sistema, e depois calculando sucessivamente termos de ordem mais alta que são agregados aos termos já existentes, funcionando como correções cada vez mais precisas à expressão inicial. Essa soma de termos é uma série de potências na constante de acoplamento. A abordagem perturbativa é possível no eletromagnetismo, cuja constante de acoplamento é aproximadamente igual a 1/137, porém é impossível na interação forte, devido ao valor extremamente alto da constante de acoplamento, aproximadamente igual a 15. (Na interação fraca a constante é da ordem de $10^{-5}$.) 
1943/1946/1948: Tomonaga - Antecipação da eletrodinâmica quântica covariante

1947: Lamb e Retherford - Experimento sobre a estrutura hiperfina do hidrogênio

1947: Bethe - Cálculo não-relativístico do Lamb shift usando renormalização de massa

1948/1949: Feynman, Schwinger - Eletrodinâmica quântica renormalizável e covariante; grande impulso ao programa da teoria quântica do campo

1949: Dyson - Equivalência dos formalismos de Feynman, Schwinger e Tomonaga; classificação sistemática dos tipos de divergências da eletrodinâmica quântica

Anos 5o: Extraordinário sucesso empírico da eletrodinâmica quântica

Em meio ao descrédito em que havia caído a teoria quântica do campo, porém, foi lançada a semente que, anos depois, permitiria o seu renascimento. Trata-se da teoria de gauge não-abeliana, proposta por C. N. Yang e R. L. Mills em 1954 (Yang \& Mills, 1997) e, independentemente, por Ronald Shaw em 1955 (Shaw, 1997) e por Ryoyu Utiyama em 1956 (Utiyama, 1997). Essa teoria viria a desempenhar um papel crucial em todo o desenvolvimento posterior do programa da teoria quântica do campo. E aqui tem início o segundo episódio no qual a renormalização viria a desempenhar um papel fundamental.

O termo "teoria de gauge" refere-se a um tipo particular de invariância ou simetria que determinadas teorias possuem. A invariância de gauge, ${ }^{8}$ no caso clássico, consiste em se poder somar ao potencial o gradiente de uma função arbitrária, e ainda assim manter inalteradas as previsões experimentais da teoria. A eletrodinâmica de Maxwell, por exemplo, é invariante de gauge clássica. No caso dos campos quânticos, a invariância de gauge consiste em se poder preservar as previsões experimentais quando se efetua, além da adição do gradiente, também uma rotação arbitrária na fase do campo. O conceito de invariância de gauge havia sido proposto por Hermann Weyl já nos primórdios da física quântica. ${ }^{9}$

8 Ocasionalmente este termo também é traduzido em português como "invariância de calibre".

9 Ver "Gravitation and electricity", de 1918 (Weyl, 1997a) e "Electron and gravitation", de 1929 (Weyl, 1997b). Sobre a origem e o desenvolvimento histórico das teorias de gauge, pode-se consultar os artigos de revisão de Jackson \& Okun (2001) e de O’Raifeartaigh \& Straumann (2000). 
A teoria de Yang e Mills se refere ao spin isotópico, que é uma quantidade conservada na interação forte. A hipótese feita por eles foi de que o spin isotópico obedeceria a uma simetria de gauge local não-abeliana, expressa pelo grupo SU(2). Uma simetria é dita global quando as equações são transformadas da mema maneira em todos os pontos do espaço-tempo, e local quando a transformação pode ser diferente em diferentes pontos. O fato de que a simetria de spin isotópico utilizada por Yang e Mills é do tipo local significa, em termos físicos, a possibilidade de transformar prótons em nêutrons, e vice-versa, de maneira independente para cada partícula, isto é, as transformações não precisam ser executadas "em uníssono", por assim dizer; além disso, o resultado de uma seqüência de transformações depende da ordem em que elas são efetuadas. $^{10}$

Uma vez imposta a invariância de gauge, Yang e Mills determinaram qual seria o campo correspondente, executaram o processo de quantização e obtiveram quanta com spin unitário e spin istópico unitário, e carga que poderia ser nula, positiva ou negativa. (Essas partículas corresponderiam aos fótons da eletrodinâmica quântica.) Duas questões acerca da teoria tiveram, porém, que ser deixadas em aberto pelos autores, devido a dificuldades técnicas: o problema da massa dos quanta do campo e a questão da renormalizabilidade. ${ }^{11}$ Por causa das dificuldades encontradas, a teoria de gauge não-abeliana não parecia ser aplicável à interação forte.

Restava ainda a possibilidade de que a interaçãofraca fosse passível de descrição por meio de uma teoria do tipo de Yang-Mills. Ademais, como a teoria de gauge era inspirada na eletrodinâmica quântica, talvez as interações fracas e eletromagnéticas fossem, em algum sentido, manifestações de uma mesma interação "eletrofraca"

10 Uma propriedade fundamental da invariância de gauge é que ela explica a existência dos campos de interação entre as partículas materiais. A relação profunda que existe entre a simetria de gauge e a existência de campos mediando as interações pode ser descrita do seguinte modo. Para descrever partículas relativísticas livres, isto é, na ausência de campos - por exemplo, elétrons com spin 1/2 - escreve-se um lagrangiano em termos de operadores que correspondem a "campos de matéria”. Para obter a invariância do lagrangiano sob transformações de fase locais dos campos de matéria, é preciso substituir as derivadas espaço-temporais ordinárias pelas chamadas derivadas covariantes. Ocorre que as derivadas covariantes incluem, por definição, potenciais de gauge, e portanto automaticamente acoplam campos de gauge aos campos de matéria. Portanto, pode-se dizer que a invariância de gauge requer a introdução de campos de interação. Assim, por exemplo, no caso da eletrodinâmica, a exigência de invariância de gauge implica a existência de um campo cujos quanta são os fótons.

11 Colocava-se o seguinte dilema: para que a simetria básica da teoria fosse preservada, os quanta do campo deveriam ter massa nula; por outro lado, sabe-se que a interação forte é de curto alcance, o que implica quanta com massa grande. O dilema se tornava mais agudo uma vez que Yang e Mills concluíram que a renormalizabilidade da teoria dependia de maneira essencial do fato de as partículas possuírem massa nula. Para complicar a situação, os próprios quanta do campo possuem spin isotópico - vale dizer, eles podem interagir entre si - o que tornava os cálculos de renormalização bastante difíceis. 
subjacente. Depois de algumas tentativas preliminares por parte dos físicos, foram propostas duas teorias de gauge unificando o eletromagnetismo e a interação fraca, por Sheldon Glashow (1961) e por Abdus Salam e John Ward (Salam \& Ward, 1964), utilizando o grupo de simetria $\mathrm{SU}(2) \times \mathrm{U}(1)$. Nessas teorias, o lagrangiano da interação previa a existência de quatro bósons: o fóton e três bósons vetoriais fracos (um com carga positiva, um negativo e um neutro). Porém havia o problema da diferença de massa, que é nula para o fóton, mas deveria ser não-nula para as outras três partículas. As massas das partículas precisavam ser inseridas "manualmente" na teoria, por assim dizer. Além disso, restava a questão de como se poderia ter bósons massivos sem destruir a invariância de gauge.

A idéia crucial que faltava para solucionar esses problemas era a quebra espontânea de simetria. Na quebra espontânea de simetria, a noção fundamental envolvida é de uma simetria que está presente no lagrangiano de forma exata mas que não se manifesta em termos físicos, ou se manifesta de forma apenas aproximada. Colocando de outro modo, enquanto a totalidade das soluções de uma equação de campo possui a simetria, uma solução particular pode não a possuir. A simetria fica "escondida" ou, como se costuma dizer, é "espontaneamente quebrada". Em 1961, Jeffrey Goldstone propôs um mecanismo para obter a quebra espontânea de simetria na teoria quântica do campo (Goldstone, 1961). ${ }^{12}$ Porém Steven Weinberg, Salam e o próprio Goldstone mostraram em 1962 que, em muitas teorias, a quebra espontânea de simetria teria que ser acompanhada pelo surgimento de partículas de spin um e massa nula, os chamados "bósons de Goldstone" (Goldstone, Salam \& Weinberg, 1962). Isso constituía um problema, uma vez que tais partículas de massa nula não foram observadas nem poderiam desempenhar um papel em interações de curto alcance.

Peter Higgs completou o mecanismo, valendo-se da invariância de gauge para mostrar como as partículas podem ganhar massa via quebra espontânea de simetria sem o surgimento de partículas de massa nula (Higgs, 1964a; 1964,b; 1966). ${ }^{13}$ Por meio

12 Goldstone considerou um campo escalar complexo cujo potencial, no plano complexo, tinha uma forma simétrica parecida com a de uma forma de bolo, com um "monte" ou máximo local na origem e todo um círculo de mínimos absolutos ao redor. $\mathrm{O}$ estado de vácuo, portanto, não era único, ou, como se diz em teoria quântica, era degenerado. Goldstone incluiu então na teoria uma "regra de superseleção" proibindo as superposições de estados construídos a partir de vácuos diferentes. (Como já mencionamos, na teoria quântica do campo os estados do campo são construídos a partir do estado de vácuo por meio de combinações de operadores de criação e aniquilação.) Assim um estado de vácuo particular deve ser escolhido - o que é feito especificando-se um ângulo no círculo de mínimos - o que destrói a simetria original.

13 Higgs considerou um lagrangiano que era uma generalização do de Goldstone, invariante sob o grupo U(1), com o mesmo campo escalar complexo, e o mesmo tipo de vácuo degenerado, porém agora incluindo também o acoplamento 
do mecanismo de Higgs desaparecem os bósons de Goldstone, os quanta de campo adquirem massa, e aparece também uma partícula massiva que é conhecida como "bóson de Higgs". O mecanismo de Higgs também funciona para teorias baseadas no grupo não-abeliano $\mathrm{SU}(2) \times \mathrm{U}(1)$, o que abriu o caminho para uma teoria de gauge unificada das interações eletromagnéticas e fracas. Essa teoria seria formulada independentemente por Weinberg, em 1967, e por Salam, em 1968 (ver Weinberg, 1967; Salam, 1994a). Eles partiram, respectivamente, das teorias de Glashow (1961) e de Salam \& Ward (1964), e usaram o mecanismo de Higgs para explicar as massas das partículas, em vez de introduzir as massas "manualmente". A teoria "eletrofraca" de WeinbergSalam não despertou interesse nos primeiros anos após sua formulação, uma vez que a teoria quântica do campo ainda atravessava uma fase de desprestígio na época. Além disso, não se tinha certeza quanto à sua renormalizabilidade. Esse cenário iria se alterar de forma dramática no início dos anos 7̧o.

Em 1971, Gerard 'tHooft conseguiu demonstrar que as teorias de gauge massivas com quebra espontânea de simetria são renormalizáveis ('tHooft, 1971a; 1971b; cf. também 'tHooft \& Veltman, 1972a; 1972b). Esta era a peça que faltava no quebra-cabeças, no plano teórico. Não só essa demonstração resgatou a teoria de Weinberg-Salam do esquecimento como levou a um renascimento do próprio programa da teoria quântica do campo. A partir de 1971, observa-se uma verdadeira explosão no número anual de citações dos trabalhos de Weinberg e Salam, saltando de zero citações por ano para centenas de citações (cf. Pickering 1984, p. 172), o que indica claramente o aumento de interesse por parte dos físicos. A partir daí, assumiu grande importância a questão dos testes experimentais da teoria eletrofraca unificada. E, de fato, seguiram-se corroborações experimentais espetaculares, como a detecção das correntes neutras (Hasert et

com um campo vetorial real sem massa. Considerando ambos os campos, obtém-se quatro graus de liberdade no total. O campo escalar complexo pode ser expandido em termos de dois campos escalares reais, um dos quais corresponde ao bóson de Goldstone. Porém existe um problema: o lagrangiano assim reescrito descreve dois campos escalares reais sem massa e um campo vetorial real com massa, o que dá cinco graus de liberdade. Como uma simples mudança de variáveis não pode alterar o número de graus de liberdade de um sistema, conclui-se que um dos campos escalares não tem significado físico e deve ser eliminado; esse seria justamente o campo correspondente ao bóson de Goldstone. A eliminação desejada é conseguida por meio de uma transformação de gauge (o chamado gauge unitário). A aplicação dessa transformação resulta num lagrangiano que possui o número correto de graus de liberdade (quatro), só que, em vez de descrever um campo escalar complexo com massa e um campo vetorial real sem massa, ele descreve agora um campo escalar real com massa e um campo vetorial real com massa. Assim, é como se o campo vetorial real tivesse "engolido" o bóson de Goldstone e absorvido o grau de liberdade a ele associado, adquirindo massa no processo. A simetria original do lagrangiano, U(1), é mantida, só que não é manifesta no espectro observável de partículas. 
$a l, 1973$ ) e a descoberta dos bósons fracos $\mathrm{W}^{-}, \mathrm{W}^{+}$, e Z (Arnison et al, 1983a; 1983b). A peça que ainda falta para completar o quadro, no plano experimental, é a detecção do bóson de Higgs. ${ }^{\mathbf{1 4}}$

Nos anos 70 e 8o, a teoria quântica do campo reassumiu o primeiro plano na física teórica, inclusive no âmbito da interação forte, com o desenvolvimento da cromodinâmica quântica (a teoria dos quarks), baseada no grupo SU(3). Houve também grande interesse em buscar uma teoria capaz de unificar todas as três unificações - eletromagnética, fraca e forte - por meio de um único grupo de gauge, como o SU(4) ou SU(5). Apesar dos grandes esforços dispendidos nessa direção, porém, essas teorias, ditas "teorias da grande unificação", não foram inteiramente bem-sucedidas. Também não se conseguiu incorporar a gravitação dentro do quadro das teorias de gauge renormalizáveis. Pode-se dizer que o principal legado dessa era na física de partículas e campos é o chamado modelo padrão, constituído pela teoria eletrofraca mais a cromodinâmica quântica, dentro da estrutura $\mathrm{U}(1) \times \mathrm{SU}(2) \times \mathrm{SU}(3)$. O modelo padrão constitui, por ora, o melhor paradigma disponível para descrever a estrutura da matéria e o comportamento dos campos. ${ }^{15}$

\section{Quadro 2. Cronologia Resumida das teorias de gauge}

1918, 1929: Weyl - Primeiras sugestões do conceito de invariância de gauge

Anos 50: Dificuldades para construir teorias quânticas renormalizáveis para as interações nucleares fraca e forte; crise do programa da teoria quântica do campo; surgimento de programas alternativos (matriz-S e álgebra de correntes)

1954: Yang e Mills - Teoria de gauge não-abeliana para o spin isotópico utilizando o grupo de simetria $\mathrm{SU}(2)$; incertezas quanto à sua renormalizabilidade

14 Para um típico relato recente de uma das frentes da busca ao bóson de Higgs, cf. por exemplo Sopczak (2002). 15 Existe um sem-número de bons textos apresentando a teoria quântica do campo de um ponto de vista técnico. Mencionaremos aqui o de Itzykson \& Zuber (1985), consideravelmente avançado, o de Mandl \& Shaw (1993), mais acessível e didático do que o primeiro, o de Bjorken \& Drell (1965) que, embora antigo, ainda tem seu valor, e o de Weinberg $(1995 ; 1996)$, extremamente avançado e de caráter enciclopédico, escrito por alguém que foi um protagonista dos acontecimentos. Sobre o desenvolvimento conceitual da teoria quântica do campo, pode-se consultar o magnífico livro de Cao (1997), bem como os artigos de revisão de Weinberg (1977) e de Cushing (1982), e o estudo sociologicamente orientado de Pickering (1984). 
RaGionalidade, GonsistênGia, RetiGulaÇão e GoerênGia...

1955/1956: Shaw, Utiyama - Desenvolvimento independente da teoria de gauge não-abeliana

1961, 1964: Glashow, Salam-Ward - Teorias de gauge unificando o eletromagnetismo e a interação fraca, utilizando o grupo $\mathrm{SU}(2) \times \mathrm{U}(1)$; problema da origem da massa das partículas

1961: Goldstone - Mecanismo para a quebra espontânea de simetria na teoria quântica do campo

1962: Weinberg-Salam-Goldstone - Problema do aparecimento de bósons de massa nula ("bósons de Goldstone")

1964/1966: Higgs - Extensão do mecanismo de Goldstone para obter partículas massivas via quebra espontânea de simetria, sem bósons de massa nula; aparecimento de um bóson residual massivo ("bóson de Higgs")

1967/1968: Weinberg, Salam - Teoria de gauge unificada das interações eletromagnéticas e fracas, utilizando o grupo $\mathrm{SU}(2) \times \mathrm{U}(1)$ e o mecanismo de Goldstone-Higgs

1971: 'tHooft - Demonstração de que as teorias de gauge massivas com quebra espontânea de simetria são renormalizáveis; renascimento da teoria de Weinberg-Salam e do programa da teoria quântica do campo

1973: Hasert et al-Detecção experimental das correntes neutras (corroboração da teoria de Weinberg-Salam)

Anos 7o e 8o: Cromodinâmica quântica (teoria da interação forte) baseada no grupo SU(3); Modelo Padrão = teoria eletrofraca + cromodinâmica quântica, com estrutura $\mathrm{U}(1) \times \mathrm{SU}(2) \times \mathrm{SU}(3)$

1983: Arnison et al-Descoberta dos bósons fracos $\mathrm{W}^{-}, \mathrm{W}^{+}$, e $\mathrm{Z}^{\circ}$ (nova corroboração da teoria de Weinberg-Salam)

Atualmente: Busca ao bóson de Higgs 


\section{A POlÊMica SOBRe A RENORMALIZAÇÃo}

A renormalização claramente desempenhou um papel fundamental no desenvolvimento da teoria quântica do campo. ${ }^{\mathbf{1 6}}$ Vimos dois momentos especialmente dramáticos nesse processo. No final dos anos 4, 0 , no bojo da teoria covariante de Feynman-SchwingerTomonaga-Dyson, a renormalização permitiu superar as dificuldades da eletrodinâmica quântica e inaugurou um período de extraordinário sucesso empírico. No início dos anos 70, com base na demonstração de 'tHooft, a renormalização possibilitou o renascimento das teorias de gauge e levou à consagração da teoria eletrofraca unificada. No período que medeia entre esses dois eventos, a renormalização foi sendo gradualmente promovida ao posto de um critério tanto de construção teórica como de escolha teórica. A demonstração de 'tHooft foi como que um ponto de inflexão desse processo. A partir dela, a renormalização passou indubitavelmente a constituir uma parte essencial da metodologia da física de partículas e campos. Atualmente, quando um novo modelo teórico é proposto, a condição de renormalizabilidade condiciona fortemente a aceitação ou rejeição desse modelo.

Esse diagnóstico é corroborado tanto por físicos como por historiadores. Por exemplo, Weinberg, um dos arquitetos da unificação eletrofraca, escreveu:

Coloquei grande ênfase na condição de renormalizabilidade... Muitos físicos discordariam dessa ênfase, e de fato, pode-se vir a descobrir que todas as teorias quânticas do campo, renormalizáveis ou não, são igualmente satisfatórias. No entanto, sempre me pareceu que o requisito da renormalizabilidade coloca exatamente o tipo de restrição de que precisamos em uma teoria física fundamental. Existem muito poucas teorias quânticas do campo renormalizáveis... Precisamos muito de um princípio-guia como a renormalizabilidade para nos ajudar a selecionar, em meio à infinita variedade de teorias quânticas do campo concebíveis, a teoria que se aplica ao mundo real (Weinberg, 1977, p. 33).

16 Cabe lembrar que, na física atual, a renormalização não é necessariamente um processo voltado para a eliminação dos infinitos. Existem renormalizações finitas, não só na teoria do campo como também, particularmente, no estudo das transições de fase. Também é digno de nota o fato de que, na teoria axiomática de campos, existe interesse em estudar as propriedades de certas teorias não-renormalizáveis. Não obstante, o aspecto histórico-metodológico que ora nos interessa diz respeito à renormalização tal como ela foi originalmente executada na eletrodinâmica e nas teorias de gauge, isto é, com o objetivo de eliminar as divergências. Por isso, é nesse tipo de renormalização que iremos nos concentrar aqui. 
Ragionalidade, Gonsistêngia, retigulação e goerêngia...

Enquanto isso, Silvan Schweber, um destacado historiador da física contemporânea, escreve:

A renormalizabilidade pode ... ser considerada como um princípio regulador, guiando a construção e a seleção de teorias dentro do quadro geral da teoria quântica do campo ... É um fato histórico que os desenvolvimentos subseqüentes da teoria quântica do campo, para além do âmbito da eletrodinâmica quântica, foram efetuados empregando o princípio de renormalizabilidade como guia ... Não seria um grande exagero afirmar que os avanços mais substanciais conseguidos na teoria quântica do campo nas últimas quatro décadas foram guiados e condicionados pelo princípio de renormalizabilidade (Schweber, 1994, p. 595, 598-9).

Hoje em dia, as técnicas de renormalização fazem parte de qualquer livro-texto sobre teoria do campo. Parece forçoso admitir, como faz um consagrado texto sobre o assunto, o de Itzykson e Zuber, que "a renormalização, em todas as ordens [de aproximação], jaz no próprio cerne da teoria quântica do campo" (Itzykson \& Zuber, 1985, p. 372).

Porém a aceitação da renormalização pela comunidade científica, ainda que maciça, sempre esteve cercada por dúvidas, e nunca se viu livre de polêmicas. Isso se deve à percepção, por parte de muitos físicos, de que a renormalização constituiria, de algum modo, um procedimento inconsistente do ponto de vista matemático e lógico. As operações envolvidas - a redefinição da massa e da carga, o apelo a quantidades infinitas inobserváveis que cancelam outras quantidades infinitas, os procedimentos de corte (cutoff) executados nas integrais divergentes, os processos de limite, os esquemas de regularização dimensional - tudo isso parecia, para aqueles cientistas (nos anos 3o a 7o mais ainda do que hoje), no mínimo duvidoso do ponto de vista lógico.

Pode-se argumentar, ademais, que a inconsistência de que se acusa a renormalização possui raízes físicas. Um dos pressupostos básicos do programa da teoria quântica do campo é que ele possui caráter local, isto é, as equações que governam a evolução de um campo num ponto do espaço-tempo devem depender somente do comportamento do campo e de suas derivadas naquele ponto. Esse pressuposto de localidade implica uma noção de interações e excitações pontuais dos campos. Isso, por sua vez, implica que, nos cálculos, é preciso levar em conta os quanta virtuais com momentos arbitrariamente altos. (Recordemos aqui a complementaridade que existe entre a posição e o momento, expressa pelo princípio de incerteza: quanto mais estreita a faixa considerada para o valor da posição, mais larga a faixa admissível para o valor do momento.) Porém a inclusão de tais processos de altas freqüências geralmente leva ao aparecimento de quantidades infinitas. Assim, as divergências ultravioletas estariam, em última análise, ligadas à localidade das interações. Ora, o uso das técnicas de 
renormalização para eliminar os infinitos tem o efeito de "borrar" ou "espalhar" espacialmente o modelo pontual e local das interações, devido à imposição de um limite sobre os momentos (o cutoff). Existe aqui, portanto, uma tensão conceitual fundamental entre uma concepção estritamente pontual de interação e uma outra concepção, característica das teorias renormalizadas, onde a interação não é perfeitamente pontual. As referências que faz T. Y. Cao a um "modelo quase pontual" (Cao, 1997, p. 205-6) revelam bem esta tensão. Nesse caso, o surgimento dos infinitos não deveria ser tomado como uma indicação das limitações intrínsecas ao ponto de vista local? A renormalização não constituiria, então, um prelúdio para o enterro definitivo do programa da teoria quântica do campo? Esta não precisaria ser substituída, finalmente, por uma teoria com interações não-locais? Eram dúvidas desse tipo que pairavam na mente dos físicos desde os anos do pós-guerra.

Cumpre lembrar que nos referimos aqui, particularmente, ao período que vai dos anos 30 até o início dos anos 7o. Hoje em dia, conversando com os físicos, percebe-se que a renormalização já é mais bem aceita do que há trinta anos. Isso se deve em parte à pesquisa em teoria axiomática de campos, que procura colocar a renormalização sobre bases matemáticas mais sólidas. Porém, independentemente de um julgamento sobre o mérito dessas investigações mais recentes, nosso interesse aqui está nas escolhas teóricas e metodológicas tal como foram feitas no período em questão, com base nos conceitos de que se dispunha na época.

É interessante considerar, sobretudo, os pronunciamentos dos físicos que, mesmo tendo participado ativamente do desenvolvimento da teoria quântica do campo, assumiram uma posição crítica com respeito à renormalização. Há aqueles como Feynman (um dos criadores da eletrodinâmica quântica moderna), que se valiam regularmente da renormalização no desenvolvimento da teoria, ao mesmo tempo que observavam que a renormalização não era inteiramente satisfatória. Numa conferência proferida no Congresso Solvay de 1961, após apresentar um panorama dos êxitos e problemas da teoria naquela época, Feynman conclui: "eu não subscrevo a filosofia da renormalização" (Feynman, 2000a, p. 162). E, ao descrever, em sua conferência Nobel de 1965 , o desenvolvimento conceitual da teoria, ele escreve:

Acredito que não haja uma eletrodinâmica quântica realmente satisfatória ... Penso - embora não tenha certeza disso - que a teoria da renormalização é simplesmente uma maneira de varrer as dificuldades com as divergências para debaixo do tapete (Feynman, 1966, p. 7०7).

Também há aqueles como Dirac (ninguém menos que um dos pais da mecânica quântica, da mecânica quântica relativística e da teoria quântica do campo), que eram 
Ragionalidade, Gonsistêngia, retigulação e goerêngia...

críticos acerbos e constantes da renormalização, argumentando que ela constitui, por si mesma, um indício de que algo está muito errado com a teoria. Dirac escreveu:

Precisamos aceitar o fato de que existe algo fundamentalmente errado com a nossa teoria da interação do campo eletromagnético com os elétrons. Por "fundamentalmente errado" quero dizer que a mecânica está errada, ou a força de interação está errada. O que está errado com a teoria é tão sério quanto aquilo que estava errado com a teoria das órbitas de Bohr ... São necessárias algumas novas equações relativísticas, e novos tipos de interações devem ser postos em jogo. Quando essas novas equações e novas interações forem imaginadas, os problemas que hoje nos confundem serão automaticamente solucionados, e não mais teremos que lançar mão de processos ilógicos como a renormalização de infinitos. Esta é um completo contra-senso em termos físicos, e eu sempre me opus a ela. Trata-se apenas uma regra prática (rule of thumb) que produz resultados. A despeito dos seus êxitos, é preciso estar preparado para abandoná-la completamente, e encarar todos os êxitos que foram alcançados usando a eletrodinâmica quântica na forma usual - com os infinitos removidos por processos artificiais - como sendo meros acidentes, nos casos em que fornecem as respostas certas, do mesmo modo que os êxitos da teoria de Bohr foram considerados meramente acidentais quando corretos (Dirac, 1983, p. $5^{3-5}$ ).

Existia (e ainda hoje existe), entre uma parcela considerável da comunidade científica, uma forte sensação de que a renormalização é em si mesma um indício de que falta algo fundamental na base conceitual da teoria quântica do campo. Não obstante, devido ao sucesso extraordinário das teorias renormalizáveis, ao mesmo tempo parece impossível escapar ao domínio da renormalização. Temos assim, em resumo, uma situação epistêmica bastante estranha: por um lado, a teoria quântica do campo foi empiricamente bem sucedida, e no entanto foi criticada; por outro lado, a renormalizacão se afigura inconsistente, e contudo foi aceita.

\section{UMA ANÁLISE RETIGULAGIONAL DA RENORMALIZAÇÃo}

Desejamos interpretar o desenvolvimento da teoria quântica do campo, e o episódio da emergência da renormalização, em termos do modelo reticulado de racionalidade científica. Esse modelo foi desenvolvido por Larry Laudan nos anos 8o, e está exposto principalmente em seu livro Science and Values (Laudan, 1984), com alguns importantes aperfeiçoamentos referentes à metodologia e à metametodologia no artigo "Progress 
or rationality? The prospects for normative naturalism" (Laudan, 1987). Creio que se trata da perspectiva mais viável e fértil de que dispomos para tratar a questão da racionalidade científica e da justificação dos padrões de avaliação em ciência. Não seria possível, dentro dos limites deste artigo, apresentar detalhadamente o modelo reticulado, de um modo que fizesse justiça à importância que ele possui dentro da atual filosofia da ciência. (Existe uma literatura filosófica considerável, surgida nos anos 80 e 90, discutindo diversos aspectos e implicações do modelo.) Por isso, vamos nos limitar a recapitular de forma muito sucinta os traços essenciais.

O modelo reticulado pressupõe que o conhecimento científico, numa dada disciplina, está estruturado em três componentes fundamentais: o das teorias [representado por T], o da metodologia [M] e o dos valores cognitivos ou axiologia [A], tal como esquematizado na Figura 1. A metodologia, em particular, é constituída não apenas pelos métodos, mas também por imperativos hipotéticos que expressam conexões entre os valores cognitivos, entendidos como fins, e os métodos, entendidos como meios para a obtenção daqueles fins. Existem relações de influência cognitiva mútua entre todos os pares de componentes [relações que podemos indicar por $\mathrm{A} \rightarrow \mathrm{T}, \mathrm{A} \leftarrow \mathrm{T}, \mathrm{A} \rightarrow \mathrm{M}, \mathrm{A} \leftarrow$ $\mathrm{M}, \mathrm{M} \rightarrow \mathrm{T}$ e $\mathrm{M} \leftarrow \mathrm{T}$, ou, abreviadamente, $\mathrm{T} \leftrightarrow \mathrm{M}, \mathrm{A} \leftrightarrow \mathrm{M} \mathrm{eA} \leftrightarrow \mathrm{T}]$. A estrutura completa do reticulado consiste, portanto, em três componentes e três pares de relações entre esses componentes.

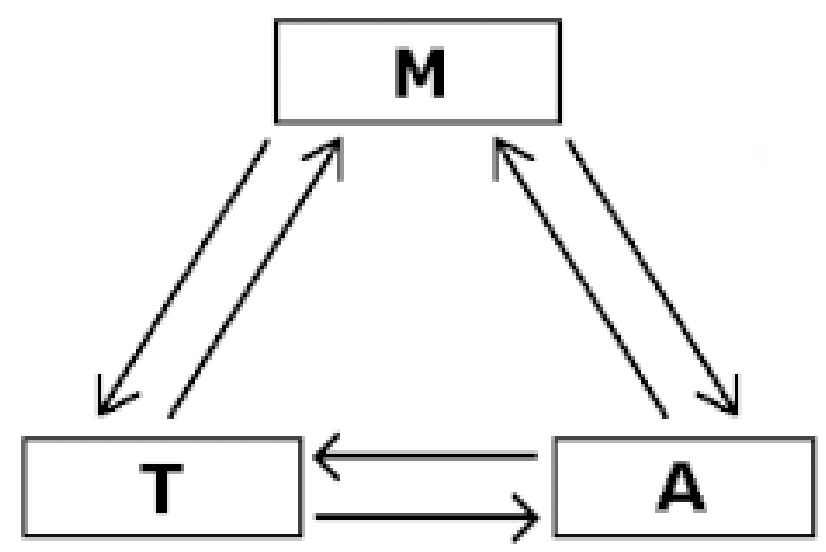

Figura 1. Estrutura do modelo reticulado. 
RaGionalidade, GonsistênGia, RetiGulaÇão e GoerênGia...

Nessa estrutura triangular, a justificação epistêmica se dá por um processo de ajuste mútuo entre os componentes do sistema. Uma escolha científica estará racionalmente justificada quando contribuir para maximizar a adequação mútua entre os componentes do reticulado. Esse processo não é hierárquico. Todos os componentes do sistema se encontram em pé de igualdade: nenhum deles tem proeminência sobre os outros. Trata-se, por assim dizer, de adequar não somente os meios aos fins [ou seja, as influências "clássicas" $\mathrm{A} \rightarrow \mathrm{T}, \mathrm{A} \rightarrow \mathrm{M}$ e $\mathrm{M} \rightarrow \mathrm{T}$ ], mas também os fins aos meios [isto é, as influências "recíprocas" $\mathrm{A} \leftarrow \mathrm{T}, \mathrm{A} \leftarrow \mathrm{M}$ e $\mathrm{M} \leftarrow \mathrm{T}$ ]. Além disso, cabe notar que nenhuma parte da metodologia ou da axiologia está imune a revisão, caso as influências provenientes do restante do sistema sejam fortes o suficiente para pressionar nesse sentido. (Em outras palavras, na perspectiva laudaniana, não existe um "núcleo duro" metodológico nem axiológico.)

O modelo reticulado prevê que as mudanças científicas, quando ocorrem, nunca afetam simultaneamente todos os componentes do sistema. Para que o processo reticulacional de ajuste mútuo entre os três componentes possa funcionar, pelo menos um componente precisa funcionar, em cada instante, como um pivô provisoriamente fixo em relação ao qual os outros se ajustam. O processo de transformação da estrutura teoria-metodologia-valores por meio de uma sucessão de transformações parciais é precisamente o que se denomina reticulação. Isso leva a um cenário gradualista de mudança científica, por oposição a um cenário holista, onde as transformações ocorreriam em todo o sistema de uma só vez. De acordo com a perspectiva reticulacional, mesmo uma mudança conceitual aparentemente "revolucionária", que parece passar de um só golpe de um complexo teoria-metodologia-axiologia para outro totalmente diferente, consiste, na realidade, em uma seqüência de mudanças parciais, locais e que, tomadas individualmente, podem ser perfeitamente racionais.

O episódio da renormalização na teoria quântica do campo pode ser sintetizado em termos reticulacionais da seguinte maneira. Consideremos primeiramente a axiologia da disciplina. Não há dúvida de que, tal como ocorre em muitas outras disciplinas científicas, a axiologia da física do campo incluía, desde o início, os valores de adequação empírica e poder preditivo. Também podemos supor que o valor da consistência matemática fazia parte, originalmente, da axiologia.

Inicialmente, com o objetivo de implementar os fins cognitivos de adequação empírica e poder preditivo, foi formulada uma certa regra metodológica de caráter geral, a saber: "se aceitamos os fins de adequação empírica e poder preditivo, então deve-se dar preferência àquelas teorias que não atribuam valores infinitos às quantidades que admitem interpretação em termos observáveis". De fato, uma teoria que prediz valores infinitos para quantidades observáveis não é capaz de fazer previsões aproveitáveis, e portanto não pode ser empiricamente adequada. Esta é uma influência da axiologia sobre a metodo- 
logia, que pode ser representada como $\mathrm{A} \rightarrow \mathrm{M}$. Sob o ponto de vista dessa regra metodológica, optar por uma teoria que atribuísse valores infinitos a quantidades observáveis seria uma escolha irracional. Desse modo a metodologia passa, por sua vez, a exercer uma pressão sobre as teorias, que pode ser abreviada como $\mathrm{M} \rightarrow \mathrm{T}$. Essas duas interações se encontram ilustradas na Figura 2.

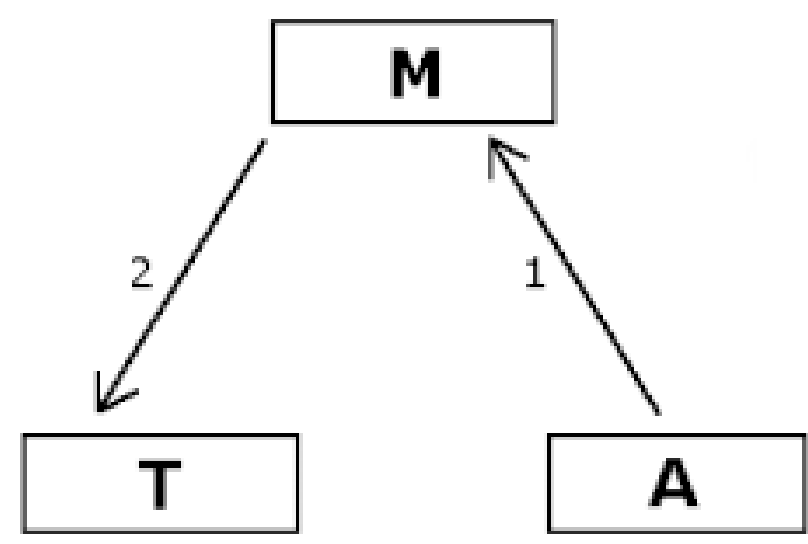

Figura 2. Interações $A \rightarrow M$ e M $\rightarrow$ T no reticulado da teoria quântica do campo.

As teorias do campo somente eram capazes de fazer jus a essa regra metodológica recorrendo a um expediente - a renormalização - que possuía, no início (anos $3 \circ$ e 4.0), caráter puramente formal e ad hoc. Por ser matematicamente não-standard, esse expediente era visto inicialmente com desconfiança. Porém esse mecanismo passou a ter o apoio de uma classe crescente de teorias altamente bem sucedidas (no final dos anos 4 , e início dos anos 50), e finalmente resultou num procedimento sistemático que, com o passar dos anos, logrou impor-se no plano metodológico. Temos aqui uma influência retroativa das teorias sobre a metodologia, interação que pode ser representada como $\mathrm{T} \rightarrow \mathrm{M}^{\prime}$ (onde a linha indica uma modificação em M), como mostra a Figura 3. 


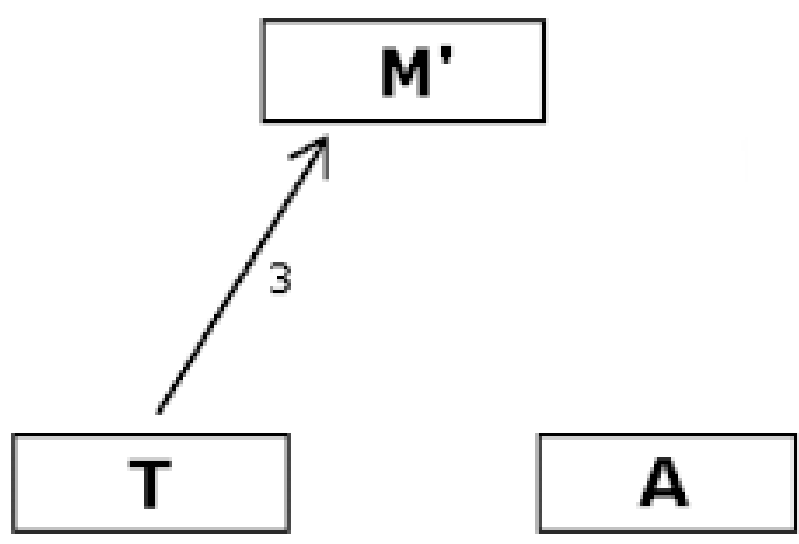

Figura 3. Interação $T \rightarrow$ M' no reticulado.

Finalmente, o procedimento de renormalização consolidou-se de tal maneira (no início dos anos 70) que se transformou num critério de formulação e escolha de teorias. Isso corresponde a uma influência que a metodologia (modificada) passa a ter sobre as novas teorias, abreviadamente $\mathrm{M}^{\prime} \rightarrow$ T', como ilustra a Figura 4 .

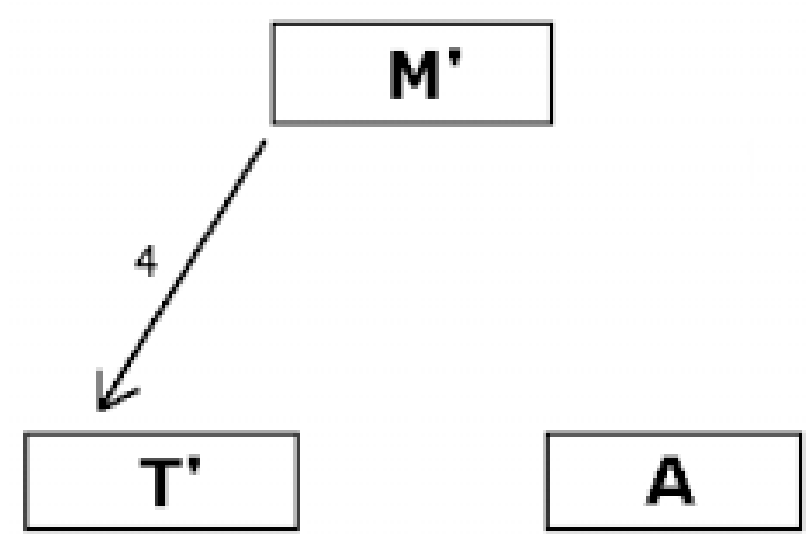

Figura 4. Interação M' $\rightarrow$ T’ no reticulado. 
É interessante notar que o estabelecimento da renormalização como parte constituinte da metodologia não se deu diretamente a partir da axiologia, isto é, pela formulação de uma regra metodológica estipulando a renormalização como um meio para o atingimento de determinado fim. Em vez disso, o processo se deu "pelo outro lado", por assim dizer - pelo lado das teorias. Quando a renormalização começou a ser aplicada, ela sequer fazia parte da metodologia da física do campo. Não estava ainda codificada em regras metodológicas. A renormalização era um procedimento que se encontrava inicialmente restrito ao âmbito das teorias, num registro formal e $a d$ hoc. Somente à medida que se foi percebendo que essa técnica formal estava estreitamente associada a uma classe de teorias bem sucedidas, é que ele foi sendo alçado à condição de critério metodológico. Assim, a renormalização não foi aceita por méritos conceituais intrínsecos, nem com base em considerações filosóficas, mas simplesmente em conseqüência de uma forte pressão cognitiva decorrente da existência de teorias que eram extremamente bem sucedidas e exibiam a propriedade de ser renormalizáveis.

O resultado dessa interação também é interessante porque a metodologia modificada (com renormalização) [M'] estava em conflito com outro fim cognitivo da axiologia [A], a saber, a consistência. Daí as críticas por parte de vários cientistas, como vimos. Porém, como sabemos, isso não foi suficiente para evitar a aceitação do procedimento de renormalização. Essa aceitação pode ser interpretada como uma modificação na axiologia, com um enfraquecimento ou suspensão temporária do valor da consistência. Temos portanto uma influência da metodologia (modificada) sobre a axiologia, abreviadamente $\mathrm{M}^{\prime} \rightarrow \mathrm{A}^{\prime}$ (onde a linha indica uma modificação em A), como se acha esquematizado na Figura 5 .

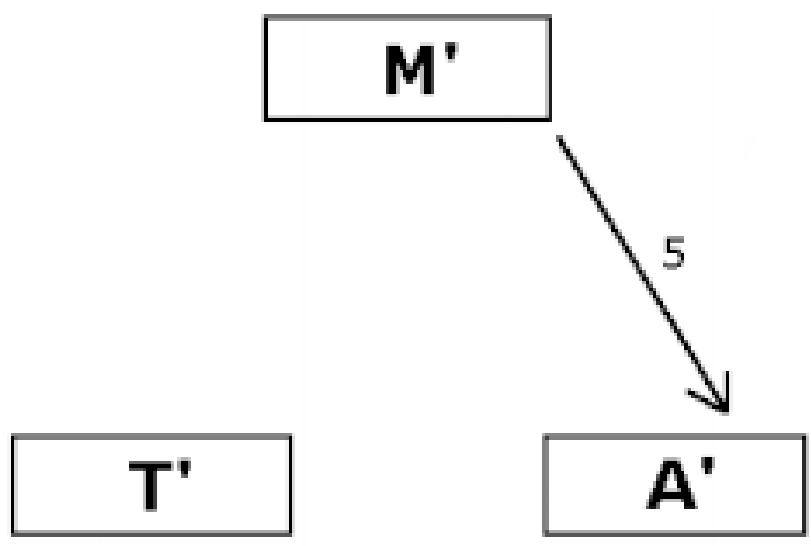

Figura 5. Interação M' $\rightarrow$ A' no reticulado. 
Ragionalidade, GonsistênGia, RetigulaÇão e Goerência...

Assim, partindo de uma determinada configuração inicial do reticulado, chegamos a uma configuração totalmente diferente, esquematizada na Figura 6. Esta nova configuração, por sua vez, poderá continuar evoluindo, por meio de novos processos de reticulação.

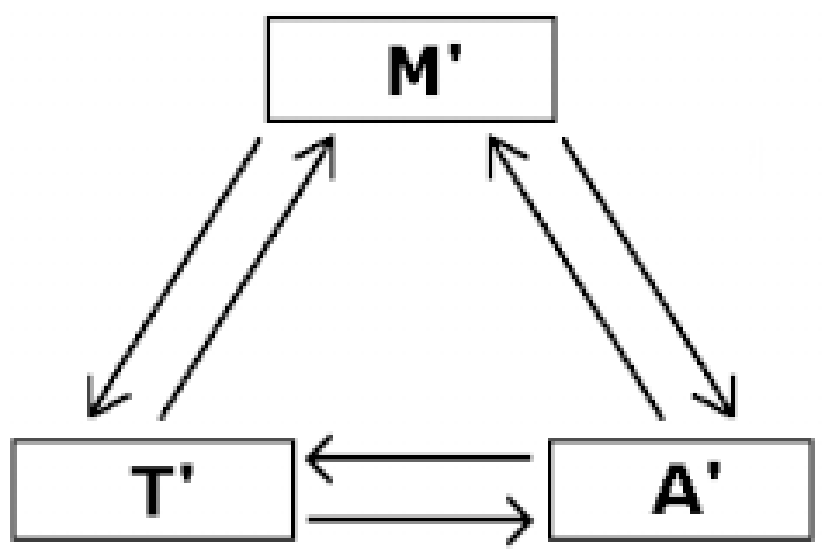

Figura 6. Configuração resultante do reticulado da teoria quântica do campo.

O caso da renormalização na teoria quântica do campo nos coloca, portanto, diante de uma situação na qual ocorrem múltiplas interações epistêmicas, não-hierárquicas, dentro do reticulado de uma disciplina. Temos um processo gradual de ajuste mútuo não somente dos meios aos fins, mas também dos fins aos meios. Em termos mais gerais, é um ajuste multidirecional entre os componentes do reticulado cognitivo da teoria do campo. Desse modo, sob a concepção instrumental de racionalidade que caracteriza a perspectiva reticulacional, pode-se afirmar que o estabelecimento da renormalização na teoria quântica do campo foi um processo racional.

\section{A questão da GonsistênGia}

Nossa análise coloca em destaque um aspecto especialmente interessante desse processo, que é o estatuto da consistência. A aceitação da renormalização e sua incorporação à metodologia permitiram aos cientistas atingir certos fins cognitivos, porém ao preço de ir contra um outro valor, que teve de ser enfraquecido na axiologia. $\mathrm{O}$ problema é que não se trata de um valor "qualquer", mas sim o valor da consistência, que 
aparentemente constitui um dos padrões mais intocáveis da Razão ocidental moderna. Um crítico racionalista poderia objetar: como pode ser racional a aceitação de um procedimento inconsistente? Como pode um relaxamento da consistência ser considerado racional? A consistência não é uma das características constitutivas da própria racionalidade? E, nesse caso, um gesto no sentido de derrogá-la não teria que ser considerado irracional?

Nossa resposta a essa crítica toma por base a tese reticulacional de que nenhuma parte de um reticulado - em particular, nenhum valor cognitivo - está, em princípio, imune a revisão. A consistência é um elemento do componente axiológico do reticulado, do mesmo modo que, por exemplo, as equações de campo ou as relações de comutação fazem parte do componente teórico. Como tal, a consistência não é um valor absoluto, e pode ser enfraquecida - talvez provisoriamente e de modo parcial - se isso for conveniente para manter a aplicabilidade de outros valores cognitivos e preservar a coerência global do reticulado. Quando se trata de preservar o princípio geral de racionalidade como adequação instrumental mútua, até mesmo um movimento no sentido de enfraquecero princípio de consistência pode ser um movimento válido, uma vez que o princípio de racionalidade tem precedência sobre qualquer outro princípio axiológico, metodológico ou teórico.

Assim, uma incompatibilidade da renormalização com o princípio de consistência não deveria ser tomado como uma indicação de que a própria noção de racionalidade é defeituosa, mas sim como uma indicação de que existe uma tensão interna no componente teórico, ou, em outras palavras, um problema conceitual. Como já haviam notado Imre Lakatos (1978a) e o próprio Laudan (1977), a existência de problemas conceituais é a regra, e não a exceção, em qualquer corpo de conhecimento científico razoavelmente desenvolvido. Nossa estratégia seria, portanto, a de entender o problema da inconsistência da renormalização como sendo um problema conceitual, em vez de um problema acerca da própria noção de racionalidade utilizada.

Resta o problema de solucionar tal inconsistência no componente teórico, ou, equivalentemente, de resolver a tensão entre o componente teórico e o valor da consistência no componente axiológico. A solução dada pela maioria dos físicos - a saber, enfraquecer o valor da consistência e apegar-se ao programa da renormalização, sem questionar o arcabouço conceitual - não parece ser uma alternativa que tenha muito apelo para os filósofos. Porém existem outras possibilidades que iriam requerer uma investigação mais profunda dos aspectos estruturais da teoria do campo. Uma possibilidade seria mostrar que a atual teoria quântica do campo pode ser subsumida a uma teoria mais geral, que seja consistente. Outra possibilidade seria substituir a teoria quântica do campo por uma teoria inteiramente diferente, fundada sobre outros pressupostos - como, por exemplo, a teoria das supercordas. Ainda outra possibilidade seria incluir a teoria quântica do campo dentro de um esquema lógico mais geral - por exem- 
plo, isolando as partes mutuamente inconsistentes da teoria e executando um processo de unificação formal por meio da lógica multidedutiva, semelhante ao que foi feito pelo lógico brasileiro Edelcio G. de Souza para o modelo atômico de Bohr (Souza, 2000). Em resumo, este não seria um problema para os teóricos da racionalidade, e sim para os especialistas em axiomática.

Desejo, em conclusão, considerar a possibilidade de uma modificação na própria lógica subjacente. Confrontados com a objeção de que a noção de racionalidade aqui empregada é insatisfatória - uma vez que, no caso da renormalização, atribui o estatuto de racional a algo que é aparentemente ilógico - poderíamos tentar elaborar uma resposta da seguinte maneira. Notemos que a objeção, tal como está formulada, implicitamente torna a racionalidade dependente da lógica. Seria legítimo, então, perguntar: qual lógica? Pressupor a lógica clássica, sem mais, seria, em muitos casos, ingênuo. As limitações da lógica clássica enquanto ferramenta para capturar algumas sutilezas da racionalidade científica já são bem conhecidas. Alguns exemplos são: a coexistência de teorias inconsistentes durante o período da antiga teoria quântica (i. e. anterior a 1925), a aceitação de noções como a complementaridade na moderna mecânica quântica, e a dificuldade de conciliar teoria quântica e relatividade geral numa escala próxima ao comprimento de Planck. Exemplos como esses mostram que não faltam dificuldades para uma racionalidade científica baseada exclusivamente na lógica clássica.

O que se sugere é que a escolha da lógica de um sistema teórico deveria ser feita numa base instrumental, tanto quanto a escolha das hipóteses científicas. Alógica não deve ser vista como parte de uma noção a priori de racionalidade: em vez disso, ela deve ser vista como uma parte do componente teórico de um reticulado. Assim, a escolha da lógica é, muito mais do que comumente se pensa, uma questão contingente: a lógica adequada para um sistema científico é um dos elementos que devem ser escolhidos de maneira a resultar numa configuração do reticulado que maximize a adequação mútua entre os componentes. Como observamos anteriormente, isso tem o efeito de deslocar o registro da questão original - e, ao mesmo tempo, também indica uma possível solução: se a renormalização conflita com aquilo que seria esperado com base na lógica clássica, isso constitui um problema conceitual para a teoria, e neste caso a solução pode estar numa mudança da própria lógica que é pressuposta.

Existem, na filosofia da ciência, precedentes para uma posição deste tipo. Vamos encontrar esses precedentes em Hilary Putnam e em W. v. O. Quine. De Putnam temos a tese do caráter contingente da lógica, defendida em seu artigo de 1969, "The logic of quantum mechanics" (Putnam, 1979a). ${ }^{17}$ Naquele texto, Putnam lembra que, no 
contexto da teoria da relatividade, a geometria não-euclidiana precisou substituir a geometria euclidiana porque esta se revelou empiricamente falsa. De modo análogo, no contexto da mecânica quântica, deduções acerca dos "enunciados empíricos elementares" da teoria, se executadas por meio da lógica clássica, levam a resultados que contradizem a experiência. Portanto, a lógica clássica precisaria dar lugar a uma lógica quântica não-distributiva (já proposta por G. Birkhoff e J. Von Neumann em 1936: ver Birkhoff \& Von Neumann, 1975). De acordo com Putnam e vários outros filósofos, a substituição da lógica clássica pela lógica quântica permitiria esclarecer diversas questões notoriamente difíceis nos fundamentos da mecânica quântica. (Cabe notar que a lógica quântica se tornou, a partir dos anos 60 e 7o, uma linha de pesquisa bastante ativa dentro da área de fundamentos da mecânica quântica.)

De Quine nos vem a tese da inexistência de uma fronteira nítida entre os enunciados da lógica e os enunciados empíricos dentro de uma rede conceitual, tese expressa em seu famoso texto de 1953, "Two dogmas of empiricism" (Quine, 1980a). Segundo Quine, a única diferença que existe entre os enunciados da lógica, os postulados teóricos e os enunciados empíricos está no fato de que estes últimos estão mais próximos da "periferia" da rede conceitual, ao passo que os primeiros estão imbricados mais profundamente, próximos ao "núcleo" do sistema. Os enunciados empíricos são, portanto, mais fáceis de serem abandonados, modificados ou substituídos, em face de um resultado experimental "anômalo", ao passo que os postulados teóricos são menos suscetíveis às mudanças, e os enunciados da lógica menos ainda. Porém, em princípio, nem mesmo estes últimos são intocáveis. Podemos imaginar uma classificação dos enunciados científicos ao longo de um espectro, com alguns mais próximos do extremo "empírico" e alguns mais próximos do extremo "formal", enquanto outros estão situados numa região intermediária. A diferença que existe entre eles é basicamente uma diferença de grau, não de natureza.

Tomados em conjunto, os argumentos de Putnam e Quine apontam na mesma direção - para uma concepção acerca da estrutura do conhecimento científico, e acerca do estatuto da lógica dentro dessa estrutura, que é plenamente compatível com a que sugerimos aqui. A lógica passa a ser vista como constituindo apenas uma parte, entre tantas outras, do reticulado - uma parte que é talvez mais difícil de modificar, mas que não deveria ser tomada a priori como sendo imutável. Assim, em uma mudança da lógica pressuposta pelo sistema talvez se possa encontrar a solução para o problema conceitual que a renormalização coloca para a física do campo. ${ }^{\mathbf{1 8}}$

18 Desejo registrar meus agradecimentos aos Professores Larry Laudan, Carlos O. Escobar, Caetano E. Plastino, Henrique Fleming e Newton da Costa por seus comentários. Agradeço também aos Profs. Claudemir R. Tossato e Walter M. Pontuschka pela assistência com a bibliografia. 
RaGionalidade, GonsistênGia, Retigulação e GoerênGia...

A linha de pesquisa aqui desenvolvida tem sua origem em minha tese de doutoramento (Bezerra, 1999). Partes do material deste artigo foram previamente expostas em comunicações apresentadas no III Encontro de Filosofia e História da Ciência do Cone Sul (Águas de Lindóia, SP, maio de 2002) e no X Encontro Nacional de Filosofia da ANPOF (São Paulo, SP, outubro de 2002).

\title{
Valter Alnis Bezerra
}

Pesquisador do Projeto Temático

"Estudos de Filosofia e História da Ciência" da FAPESP, pós-doutorando do Departamento de Filosofia

da Universidade de São Paulo.

bezerra@usp.br

\begin{abstract}
A study of the development of quantum field theory, in the period that goes from 1927 to 1971 , highlights the fact that renormalization techniques played a fundamental role during the whole process. In particular, renormalization rehabilitated quantum electrodynamics in the late 4 .os and, later, also rehabilitated quantum field theory as a whole and helped consolidating gauge theories in the early zos. The success of renormalization was such that, from its beginnings as a mere $a d$ hoc theoretical device, it gradually earned the condition of a criterion for the construction and appraisal of theories in field physics. One of the aims of this article is to show that this change in the methodological status of renormalization can be understood in the context of Larry Laudan's reticulational model of rationality. In spite of the extraordinary theoretical and empirical progress achieved, however, there were always polemics regarding the place that renormalization should occupy within the conceptual structure of the discipline. These polemics revolve around the fact that renormalization apparently goes against a cognitive value that is regarded as fundamental, namely, consistency. The second aim of this paper is to show how the reticulational model helps to throw light upon this question as well. We attempt to show in which sense it was rational to accept renormalization in particle and field physics despite the problem of inconsistency. To this end we make use of the theses of the reticulational model, supplemented with theses from Putnam and Quine, and from the coherence theory of justification.
\end{abstract}

KEYWORDS • Scientific methodology. Scientific rationality. Reticulational model. Cognitive values. Quantum field theory. Gauge theory. Renormalization. Inconsistency.

\section{REFERÊNGIAS BIBLIOGRÁFICAS}

NotA BibliográficA. Estão disponíveis alguns volumes de reprints fotográficos que são muito convenientes por reunir vários artigos clássicos em um único lugar, no formato em que foram originalmente publicados (inclusive com a paginação original). Em Schwinger (ed., 1958) estão reproduzidos os seguintes artigos: Bethe, 1947; Dirac, 1927; Dyson, 1949a; 1949b; Feynman, 1949a; 1949b; Jordan \& Wigner, 1928; Lamb \& Retherford, 1947; Schwinger, 1949b; 
Tomonaga, 1946; 1948; Weisskopf, 1939; 1994b. Em Feynman (2000b) encontram-se reproduzidos os seguintes: Feynman, 1948a; 1948b; 1949a; 1949b; 1966; 2000a. Finalmente, em Salam (1994b), acham-se reproduzidos os seguintes artigos: Goldstone, Salam \& Weinberg, 1962; Salam, 1994a; Salam \& Ward, 1964.

ARNISON, G. et al. "Experimental observation of isolated large transverse energy electrons with associated missing energy at $\sqrt{s}_{s}=54,0 \mathrm{GeV}$." In: Physics Letters, 122B, 1983a, p. 103-16.

"Experimental observation of lepton pairs of invariant mass around $95 \mathrm{GeV} / \mathrm{c}^{2}$ at the CERN SPS collider." In: Physics Letters, 126B, 1983b, p. 398-410.

BETHE, H. A. "The electromagnetic shift of energy levels." In: Physical Review, 72, 1947, p. 339-41.

BEZERRA, V. A. Estruturas em busca do equilíbrio: o lugar da metametodologia e o papel da coerência no modelo reticulado de racionalidade científica. Tese de doutoramento. São Paulo, Departamento de Filosofia da Universidade de São Paulo, 1999.

BIRKHOFF, G. \& VON NEUMANN, J. “The logic of quantum mechanics.” In: HOOKER, C. A. (ed.). The logico-algebraic approach to quantum mechanics. Volume I: historical evolution. Dordrecht, D. Reidel, 1975, p. 1-26.

BJORKEN, J. D. \& DRELL, S. D. Relativistic quantum fields. Nova Iorque, McGraw-Hill, 1965.

BORN, M.; HEISENBERG, W. \& JORDAN, P. “On quantum mechanics II.” In: VAN DER WAERDEN, B. L. (ed.). Sources of quantum mechanics. Nova Iorque, Dover, 1968, p. 321-85.

BROWN, L. M. \& HODDESON, L. (eds.). The birth of particle physics. Cambridge, Cambridge University Press, 1983.

CAO, T. Y. Conceptual developments of 2oth century field theories. Cambridge, Cambridge University Press, 1997 .

CUSHING, J. T. "Models and methodologies in current theoretical high-energy physics." In: Synthese, $5^{\circ}, 1982$, p. $5^{-101 .}$

DANCOFF, S. M. "On radiative corrections for electron scattering." In: Physical Review, 55, 1939, p. $959-63$.

DIRAC, P. A. M. “The quantum theory of the emission and absorption of radiation.” In: Proceedings of the Royal Society, A114, 1927, p. 24,3-65.

"The origin of quantum field theory." In: BROWN, L. M. \& HODDESON, L. (eds.). The birth of particle physics. Cambridge, Cambridge University Press, 1983, p. 39-55.

"Theory of the positron." In: MILLER, A. I. (ed.). Early quantum electrodynamics: a source book. Cambridge, Cambridge University Press, 1994a, p. 136-44.

"Discussion of the infinite distribution of electrons in the theory of the positron." In: MILLER, A. I. (ed.). Early quantum electrodynamics: a source book. Cambridge, Cambridge University Press, 1994 b, p. $145^{-} 5^{6}$.

DYSON, F. J. "The radiation theories of Tomonaga, Schwinger, and Feynman." In: Physical Review, 75, 194.9a, p. 486-502.

. "The $S$ matrix in quantum electrodynamics." In: Physical Review, 75, 1949b, p. 1736-55.

FEYNMAN, R. P. "A relativistic cut-off for classical electrodynamics." In: Physical Review, 74, 194.8a, p. $939-4.6$.

"Relativistic cut-off for quantum electrodynamics." In: Physical Review, 74, 194,8b, p. 14,30-8.

"Space-time approach to quantum electrodynamics." In: Physical Review, 76, 1949a, p. 769-89.

"The theory of positrons." In: Physical Review, 76, 1949b, p. 749-59.

"The development of the space-time view of quantum electrodynamics." In: Science, $15^{3}, 3737$, 1966, p. 699-708. 
Ragionalidade, Gonsistêngia, retigulação e goerêngia...

FEYNMAN, R. P. “The present status of quantum electrodynamics.” In: Selected papers of Richard Feynman (with commentary). Singapura, World Scientific, 2000a, p. 134-72.

Selected papers of Richard Feynman (with commentary). Ed. de L. M. Brown. Singapura, World Scientific, 200ob.

FURRY, W. \& OPPENHEIMER, J. R. "On the theory of the electron and positive." In: Physical Review, 45, 1934, p. $245^{-62 .}$

GLASHOW, S. L. "Partial-symmetries of weak interactions.” In: Nuclear Physics, 22, 1961, p. 579-88.

GOLDSTONE, J. "Field theories with 'superconductor' solutions." In: Nuovo Cimento (New series), 19, 1961, p. 154-64.

GOLDSTONE, J.; SALAM, A. \& WEINBERG, S. "Broken symmetries." In: Physical Review, 127, 1962, p. $965^{-}-70$.

HASERT, F. J. et al. "Observation of neutrino-like interactions without muon or electron in the Gargamelle neutrino experiment.” In: Physics Letters, 46B, 1973, p. 138-4, 0.

HEISENBERG, W. "Remarks on the Dirac theory of the positron." In: MILLER, A. I. (ed.). Early quantum electrodynamics: a source book. Cambridge, Cambridge University Press, 1994, p. 169-87.

HIGGS, P. W. "Broken symmetries, massless particles and gauge fields." In: Physics Letters, 12, 1964a, p. $132-3$.

. "Broken symmetries and the masses of gauge bosons." In: Physical Review Letters, 13, 1964b, p. 5०8-9.

. "Spontaneous symmetry breakdown without massless bosons." In: Physical Review, 145, 4, 1966, p. $1156-63$.

HOOKER, C. A. (ed.). The logico-algebraic approach to quantum mechanics. Volume I: historical evolution. Dordrecht, D. Reidel, 1975 .

ITZYKSON, C. \&ZUBER, J.-B. Quantum field theory. Nova Iorque, McGraw-Hill, 1985.

JACKSON, J. D. \& OKUN, L. B. "Historical roots of gauge invariance." In: Reviews of Modern Physics, $7^{3}$, 2001, p. 663-80.

JORDAN, P. \& WIGNER, E. “Über das Paulische Äquivalenzverbot.” In: Zeitschrift für Physik, 47, 1928, p. $631-51$.

JORDAN, P. \& PAULI, W. “Zur Quantenelektrodynamik ladungsfreier Felder.” In: Zeitschrift für Physik, 47,1928, p. 151- $7^{3}$.

KRAMERS, H. "Die Wechselwirkung zwischen geladenen Teilchen und Strahlungsfeld.” In: Nuovo Cimento (Série antiga), 15, 1938, p. 108-14.

LAKATOS, I. "Falsification and the methodology of scientific research programmes." In:

The methodology of scientific research programmes. Cambridge, Cambridge University Press, 1978a, p. 8-101.

. The methodology of scientific research programmes. Ed. de J. Worrale \& G. Currie. Cambridge, Cambridge University Press, $1978 \mathrm{~b}$.

LAMB, W. E. \& RETHERFORD, R. C. "Fine structure of the hydrogen atom by a microwave method." In: Physical Review, 72, 1947, p. 241-3.

LAUDAN, L. Progress and its problems: towards a theory of scientific growth. Londres, Routledge \& Kegan Paul, 1977.

Science and values: the aims of science and their role in scientific debate. Berkeley, University of California Press, 1984.

"Progress or rationality? The prospects for normative naturalism." In: American Philosophical Quarterly, 24, 1987, p. 19-31.

MANDL, F. \& SHAW, G. Quantum field theory (Revised edition). Chichester, John Wiley \& Sons, 1993. 
MILLER, A. I. (ed.). Early quantum electrodynamics: a source book. Cambridge, Cambridge University Press, 1994.

OPPENHEIMER, J. R. "Note on the theory of the interaction of field and matter." In: Physical Review, 35 , 1930a, p. 461-77.

. "On the theory of electrons and protons." In: Physical Review, 35, 193ob, p. 562-3.

O'RAIFEARTAIGH, L. (ed.). The dawning of gauge theory. Princeton, Princeton University Press, 1997.

O'RAIFEARTAIGH, L. \& STRAUMANN, N. "Gauge theory: historical origins and some modern developments." In: Reviews of Modern Physics, 72, 1, 2000, p. 1-23.

PAULI, W. \& FIERZ, M. "Theory of the emission of long-wave light quanta." In: MILLER, A. I. (ed.). Early quantum electrodynamics: a source book. Cambridge, Cambridge University Press, 1994, p. $227-4,3$.

PICKERING, A. Constructing quarks: a sociological history of particle physics. Chicago, University of Chicago Press, 1984 .

PUTNAM, H. “The logic of quantum mechanics.” In: Mathematics, matterand method: philosophical papers, volume I (Second edition). Cambridge, Cambridge University Press, 1979a, p. 174- 97.

. Mathematics, matter and method:philosophical papers, volume I (Second edition). Cambridge, Cambridge University Press, 1979b.

QUINE, W. v. O. "Two dogmas of empiricism.” In: From a logical point of view: nine logico-philosophical essays (Second, revised edition). Cambridge (Massachusetts), Harvard University Press, $1980 a$, p. $20-46$.

. From a logical point of view: nine logico-philosophical essays (Second, revised edition). Cambridge (Massachusetts), Harvard University Press, 1980b.

SALAM, A. "Weak and electromagnetic interactions." In: Selected papers of Abdus Salam (with commentary). Singapura, World Scientific, 1994a, p. 244-54.

. Selected papers of Abdus Salam (with commentary). Ed. de A. Ali; C. Isham; T. Kibble \& Riazuddin. Singapura, World Scientific, 1994b.

SALAM, A. \& WARD, J. G. "Electromagnetic and weak interactions." Physics Letters, 13, 1964, p. $168-{ }_{-11 .}$

SCHWEBER, S. S. QED and the men who made it: Dyson, Feynman, Schwinger, and Tomonaga. Princeton, Princeton University Press, 1994.

SCHWINGER, J. “Quantum electrodynamics. I. A covariant formulation.” In: Physical Review, 74, 1948, p. $1439-61$.

. "Quantum electrodynamics. II. Vacuum polarization and self energy." In: Physical Review, 75, 1949a, p. $65^{1-79}$.

. "Quantum electrodynamics. III. The electromagnetic properties of the electron - Radiative corrections to scattering." In: Physical Review, 76, 1949b, p. 790-817.

"Preface." In: SCHWINGER, J. (ed.). Selected papers on quantum electrodynamics. Nova Iorque,

Dover, $195^{8}$, p. vii-xvii.

(ed.). Selected papers on quantum electrodynamics. Nova Iorque, Dover, $195^{8 .}$

SHAW, R. "Invariance under general isotopic spin transformations." In: O'RAIFEARTAIGH, L. (ed.). The dawning of gauge theory. Princeton, Princeton University Press, 1997, p. 200-7.

SOPCZAK, A. "Higgs physics at LEP-1." In: Physics Reports, 359, 2002, p. 169-282.

SOUZA, E. G. "Multideductive logic and the theoretic-formal unification of physical theories." In: Synthese, 125, 2000, p. 253-62.

'tHOOFT, G. "Renormalization of massless Yang-Mills fields." In: Nuclear Physics, B33, 1971a, p. 173-99. "Renormalizable lagrangians for massive Yang-Mills fields." In: Nuclear Physics, B35, 1971b, p. $167-88$. 
RaGionalidade, GonsistênGia, Retigulação e GoerênGia...

'tHOOFT, G. \& VELTMAN, M. "Regularization and renormalization of gauge fields." In: Nuclear Physics, B44, 1972a, p. 189-213.

'tHOOFT, G. \& VELTMAN, M. "Combinatorics of gauge fields." In: Nuclear Physics, B50, 1972b, p. $318-53$.

TOMONAGA, S. "On a relativistically invariant formulation of the quantum theory of wave fields." In: Progress of Theoretical Physics, 1, 2, 1946, p. 27-42.

. "On infinite field reactions in quantum field theory." In: Physical Review, 74, 1948, p. 224.

UTIYAMA, R. "Invariant theoretical interpretation of interaction." In: O'RAIFEARTAIGH, L. (ed.). The dawning of gauge theory. Princeton, Princeton University Press, 1997, p. 213-39.

VAN DER WAERDEN, B. L. (ed.). Sources of quantum mechanics. Nova Iorque, Dover, 1968.

WEINBERG, S. “A model of leptons.” In: Physical Review Letters, 19, 21, 1967, p. 1264-66.

"The search for unity: notes for a history of quantum field theory." In: Daedalus, 106, 2, 1977,

p. $17-35$.

. The quantum theory of fields. Volume I: foundations. Cambridge, Cambridge University Press, 1995.

The quantum theory of fields. Volume II: modern applications. Cambridge, Cambridge University Press, 1996.

WEISSKOPF, V. "On the self-energy and the electromagnetic field of the electron." In: Physical Review, 56,1939, p. $72-85$.

“The self-energy of the electron.” In: MILLER, A. I. (ed.). Early quantum electrodynamics: a source book. Cambridge, Cambridge University Press, 1994a, p. 157-68.

. "The electrodynamics of the vacuum based on the quantum theory of the electron." In: MILLER,

A. I. (ed.). Early quantum electrodynamics: a source book. Cambridge, Cambridge University Press, 1994, b, p. 206-26.

WEYL, H. "Gravitation and electricity." In: O'RAIFEARTAIGH, L. (ed.). The dawning of gauge theory. Princeton, Princeton University Press, 1997a, p. 24-37.

“Electron and gravitation.” In: O'RAIFEARTAIGH, L. (ed.). The dawning of gauge theory. Princeton,

Princeton University Press, 1997b, p. 121-44.

YANG, C. N. \& MILLS, R. L. “Conservation of isotopic spin and isotopic gauge invariance." In:

O’RAIFEARTAIGH, L. (ed.). The dawning of gauge theory. Princeton, Princeton University Press, 1997, p. 186-96. 